\section{Identidad y nacionalismo. Una mirada sobre la búsqueda de identidad y nuevas tendencias en el diseño de modas}

María Florencia Bertuzzi ${ }^{\star}$ y Daniela Escobar ${ }^{\star *}$

\begin{abstract}
Resumen: Hablar de la situación en la que el mundo se encuentra actualmente resulta contradictorio. Por un lado, es innegable la masificación que produce la globalización, por tanto, la unidad existente entre los distintos países y las nuevas formas de comunicación y acceso a la información posibilitan la conexión instantánea a nivel global; mientras que por otro lado hay una enorme necesidad de diferenciación y, en este sentido, la vuelta a las raíces y la exploración dentro del territorio de origen han dado un nuevo sentido a la moda y le ha otorgado un nuevo rol al diseñador de indumentaria. La búsqueda de una identidad marcada se genera a partir de la reivindicación de técnicas textiles ancestrales, experimentación textil, sustentabilidad, slow fashion y upcycling, nacionalismo a flor de piel, funcionalidad de la mano con textiles inteligentes, entre otras tendencias en materia de diseño de indumentaria.
\end{abstract}

Palabras clave: moda - tendencias - Diseño de indumentaria - moda consciente - sustentabilidad - nuevo lujo - resiliencia.

[Resúmenes en inglés y portugués en las páginas 126-127]

${ }^{(*)}$ Diseñadora Textil y de Indumentaria (UP, 2012). Desde el año 2013 se desempeña como docente universitaria (UP y UNNOBA). Cursó el Programa de Formación y Capacitación Docente (UP). Actualmente se encuentra cursando un Posgrado de Especialización en Docencia Universitaria (Unnoba). Participa como coordinadora de una feria de diseño en Junín, Buenos Aires y se encuentra desarrollando un proyecto de lencería de diseño independiente.

${ }^{(*)}$ Diseñadora Textil y de indumentaria (UP, 2011). Asesora de imagen personal y corporativa (COE, 2009). Cursó el Programa de Formación y Capacitación Docente (UP, 2014) Profesora de la Universidad de Palermo en el Área de Moda y Tendencias de la facultad de Diseño y Comunicación.

Desde el año 2011 se desempeña como diseñadora de indumentaria y modelista independiente. Participa activamente del diseño de vestuario para cortos independientes. Actualmente dirige su propia marca de Diseño de Alta Costura. Forma parte del Equipo de Evaluación de Proyectos de Graduación y el Cuerpo Académico de Tutorías de la Facultad de Diseño y Comunicación de la Universidad de Palermo. 


\section{Introducción: un acercamiento a la moda argentina}

La moda ha dejado de ser tal y como se la conocía. Ha perdido en gran parte su carácter hegemónico y los centros productores de moda y tendencia, aquellas ciudades del primer mundo que no admitían ningún tipo de competencia, hoy se deben enfrentar a propuestas creativas que surgen de lugares anteriormente impensados. Es el caso de Argentina, quien se ha impuesto tanto en la región -junto a Brasil y Colombia- como a nivel global, como un laboratorio de tendencias a partir del desarrollo de indumentaria con una marcada identidad despegándose por primera vez en la historia de los mandatos europeos.

El caso argentino resulta interesante de analizar ya que desde los tiempos de la colonia la moda reflejaba la heredada cultura europea. Desde aquel entonces, los argentinos han creído que la industria nacional era de baja calidad en relación a las casas de moda extranjeras, de la misma manera se ha relacionado por décadas a la indumentaria importada con productos de lujo. En este sentido, el desarrollo de indumentaria en el país siempre ha mirado al viejo continente, ya por el año 1920 las mujeres adineradas viajaban a Europa para traer sus vestidos de París o, en caso de no poder hacerlo, los recibían a través de comisionistas que se dedicaban a traer vestidos al país para poder vendérselos a las mujeres porteñas de clase alta.

La moda en la Argentina siempre ha estado vinculada al sentir europeo que durante mucho tiempo se hizo parte de la identidad nacional, no porque el argentino no valore sus raíces autóctonas, sino porque a raíz de las inmigraciones provenientes de Europa hasta mediados del siglo XX, la población criolla ha incorporado gran parte de la cultura del viejo continente y, por lo tanto, se siente parte de ella. Esta situación, sencillamente, se puede observar, entre otras cosas, en las formas vestimentarias tanto de las mujeres como de los hombres argentinos.

Según un informe acerca del perfil migratorio de la Argentina realizado por la Organización Internacional para las Migraciones -OIM- (2008):

La inmigración transoceánica que tuvo lugar a partir de mediados del siglo XIX contribuyó de manera esencial al poblamiento del país, aportando en el período 1881-1914 algo más de 4.200.000 personas. De entre ellos, las comunidades predominantes fueron: italiana (2.000.000), española (1.400.000), francesa (170.000) y rusa (160.000). Las corrientes más numerosas se manifestaron antes de la primera guerra mundial; tal es así que en 1914 el stock de inmigrantes en el país alcanzó su máximo nivel histórico, en términos relativos, con un impacto del 30\% en el total de la población (p. 7).

Luego de la década del 60', ya en pleno proceso de globalización -o mundialización-y con una marcada economía capitalista, Argentina empieza a considerar y a consumir también la moda proveniente de Estados Unidos, que ya se imponía como una potencia mundial y como un nuevo centro productor de modas y tendencias.

El sistema productivo de indumentaria en nuestro país, hasta hace algunas décadas, se ha caracterizado por ser imitativo, ha copiado y replicado vestimentas de los diseñadores más reconocidos a nivel internacional para obtener productos capaces de competir en el 
mercado local, aunque no a nivel mundial. De alguna manera, las marcas nacionales, hasta aquel momento, no tomaban conciencia de que dentro de su propio territorio podrían encontrar los recursos necesarios para proyectarse al mundo con sus proyectos creativos. Por este motivo en la década de 1980, a partir del otorgamiento de unos créditos italianos para la fabricación de indumentaria, tal y como lo menciona Susana Saulquin en una entrevista otorgada al diario La Nación (2009), se decide crear la carrera de Diseño de indumentaria y Textil en la Universidad de Buenos Aires (UBA) en abril de 1989. Con esto se pretendía comenzar a generar moda y diseño con impronta nacional y alejarse de la copia de productos extranjeros. Si bien la intención era más que interesante, durante la década del 90' con la política económica del 1 a 1, el gobierno abrió las importaciones y el diseño argentino nuevamente quedó relegado a merced de un mercado muy competitivo en el cual la industria nacional no encontraba su lugar.

Con la crisis económica y política que el país afrontó en el año 2001, la cual se considera un antes y un después en relación al diseño argentino, el mercado comenzó a fluctuar producto del contexto político, social y económico que se vivía por aquellos tiempos y se reavivó el espíritu emprendedor de la mano de jóvenes diseñadores muy creativos. Dicha crisis catapultó al diseño argentino hacia el mundo, integrándolo en el contexto global de la moda.

El advenimiento del diseño de autor y cierta revalorización de lo artesanal ocurrido a partir del año 2000 en nuestro país abrió las puertas de una nueva cultura apoyada en la relectura de técnicas, materiales, e identidades, dormidas y marginadas frente al dictado de la producción masiva y el global star system del diseño. El resultado de ello ha sido, con mayor o menor suerte, una variedad de diseños, que oscilan entre las actuaciones más tecnológicas y las más creativas, y en los que conviven aquellos desarrollos de fuerte impronta identitaria y local, muchas veces mixturados con una cultura artesanal, junto a proyectos de base industrial y tech, ambos con vocación de proyectarse más allá de las estrechas fronteras nacionales (Lebendiker en Mercado de Industrias Creativas de Argentina, 2017, s/d).

En este sentido, coincidiendo con el pensamiento de Laureano Mon (2013) es posible determinar que la imaginación y la creatividad, más que las grandes inversiones de capital, son el ADN del diseño nacional. La clave ha sido, y sigue siendo, generar productos creativos en los cuales se fusionen características del contexto local con la tan anhelada estética global a fin de obtener indumentaria competitiva a nivel internacional.

De esta manera, cabe destacar que la moda como tal no es inherente de la cultura, la sociedad y el contexto en el que se encuentre. Frédéric Godart (2012) asegura que "La moda es un hecho social total, ya que es simultáneamente artístico, económico, político, sociológico... y afecta el tema de la expresión de la identidad social" (p. 17). En tanto, resulta ilógico pensar en la moda argentina sin analizar el recorrido que ha tenido que atravesar para, por fin, lograr que la indumentaria conocida en la actualidad como de autor, exprese el bagaje cultural, social, económico y también político que compone la identidad argentina, la cual se vincula más con la diversidad y la mezcla cultural que caracteriza a los habitantes del territorio. 


\section{Piensa global, actúa local: diseño argentino para el mundo}

Como se ha planteado anteriormente, pensar la moda como un hecho aislado del entorno es algo ilógico, por lo tanto, resulta de interés analizar brevemente el contexto actual en el cual se producen las tendencias y surge la indumentaria que, en algunos casos se convierte en moda y en otros, sin embargo, se presenta como una herramienta o un mensaje a través del cual es posible comunicar la identidad, ya sea del diseñador o de una nación.

Hablar del contexto actual requiere de un análisis profundo, los medios masivos y tecnológicos impulsados hace ya varias décadas son, en gran parte, los protagonistas de la era digital, la cual predispone cambios en las formas de comunicación principalmente, pero también en la estructuración del trabajo -como el trabajo colaborativo, el co-diseño y el networking-, permitiendo nuevas maneras de relacionarse y de llevar a cabo proyectos en cualquier parte del mundo.

Es evidente que, en este mundo globalizado, fluctuante y con cambios constantes, la moda de masas ha encontrado su contexto ideal en tanto le permite renovarse y expandirse al mundo, pero el diseño con identidad, alejado de las tendencias imperantes, se ha podido mantener en el mercado a través de propuestas que apuestan a la creatividad y a la diferenciación en un mundo que pretende la unidad.

La situación actual que vive la humanidad se caracteriza por un proceso acelerado de cambios: caída de referentes históricos, reordenamiento del mundo, el tiempo y el espacio, provocados por la globalización; búsqueda de nuevos paradigmas, estructuración de una cultura planetaria articulada a las necesidades del mercado que niega o instrumentaliza la diversidad y la diferencia (Guerrero Arias, 2002, p. 15).

Hablar de globalización implica, como plantea Colzani (2014), una contradicción, en tanto que sugiere un contexto mundialmente unificado que se caracteriza principalmente por la búsqueda imperiosa de diferenciación por parte de los individuos. Y en esta búsqueda, la vuelta a las raíces, el mirar hacia adentro o al entorno más cercano, resulta ser una buena estrategia de diferenciación entre pares y, sobre todo, una herramienta muy interesante para los diseñadores emergentes. Durante décadas el entorno cercano, los recursos que proveía el territorio y las técnicas textiles ancestrales no se habían tenido en cuenta para el desarrollo de indumentaria en el país, teniendo en cuenta que, haciendo una retrospectiva de la moda en Argentina, es posible determinar que la identidad se buscaba en torno a las raíces europeas.

Cabe destacar que a menudo se vincula el concepto de identidad con lo autóctono, con lo meramente nacional y, si se realiza un análisis de la identidad argentina, es posible determinar que no necesariamente está vinculada con lo anteriormente mencionado. Precisamente el diseño argentino parece estar más en sintonía con el lema contemporáneo "piensa global y actúa local", teniendo en cuenta que fusiona de manera efectiva técnicas industriales y artesanales en su producción, mixturando el sentir europeo con lo netamente autóctono, principalmente en relación a las materialidades y técnicas de revalorización textil artesanales. 
Como nuevas formas sustentables de pensar la producción que se apartan de la masividad, resulta interesante considerar el desarrollo de las habilidades locales, por ejemplo, la confección de artesanías y el uso de los materiales de cada lugar para comercializarlos globalmente ("glocalización”).

En el caso especial de Argentina, se abren oportunidades a partir del reconocimiento cada vez más importante de los camélidos: llamas, guanacos y vicuñas, que aportan autenticidad y tradición cultural como nuevo valor" (Saulquin, 2014, p. 46).

En este contexto, globalizado y con una inmensa necesidad de mantenerse actualizado constantemente, las nuevas formas de comunicación han jugado un papel importante en torno al desarrollo de nuevos proyectos creativos y emprendimientos. Ya no resulta imprescindible estar presente físicamente para entablar relaciones laborales o emprender nuevos proyectos, en tanto los avances tecnológicos han hecho posible nuevas estructuras de negocios que permiten estar conectados y comunicados a kilómetros de distancia pudiendo trabajar colaborativamente y poniendo en valor a todos los participantes de la cadena de producción, incluso trabajando conjuntamente entre diseñadores (co-diseño) o con personas pertenecientes o descendientes de los pueblos originarios implementando técnicas textiles artesanales para sus diseños (cooperativismo) a partir de los principios del comercio justo.

Hoy en día, sigue estando muy arraigada la creencia, tanto en diseñadores como en consumidores de moda y usuarios, de que la moda con impronta o identidad nacional consta de una traducción o copia literal de la vestimenta o diseños folclóricos representativos de la cultura étnica de un país. Esta premisa no sólo resulta antigua, sino que otorga una mirada parcializada sobre el proceso de diseño que implica no sólo el análisis histórico, la evaluación de materias primas autóctonas (y/o presentes en el territorio), el análisis y aplicación de tendencias mundiales latentes (no estacionales, sino a nivel macro ya que se involucran procesos éticos, sustentables y políticos) para generar un proceso de hibridación en relación a la identidad de cada autor determinado.

Es decir, que la toma de partido hacia una moda nacionalista no responde simplemente a cumplir con una tendencia boho o hippie chic, sino que engloba una inquietud más profunda en relación a varios aspectos a tener en cuenta.

En primer lugar, como se ha mencionado anteriormente, el análisis histórico del ambiente que se habita resulta muy enriquecedor en cuanto a las tipologías vestimentarias utilizadas en el país, así como también los accesorios utilizados y, en concordancia, se pueden explorar diferentes materias primas y técnicas de manufacturación artesanal, como así también técnicas ancestrales de adorno u ornamentación. Si se estudia específicamente al territorio argentino, se encuentran numerosas materialidades textiles muy ricas para la producción de indumentaria, calzado, accesorios y diversos objetos del vestir.

Según Henrik Kuffner (asesor técnico en fibras naturales para el proyecto "Mejora de las Economías Regionales y Desarrollo Local” ejecutado por el Instituto Nacional de Tecnología Industrial -INTI- con el apoyo de la Unión Europea) en relación a las fibras naturales argentinas, existen dos tendencias mundiales que favorecen el posicionamiento argentino en el escenario de la moda: 
Por un lado, la búsqueda de originalidad que manifiestan los consumidores se profundiza. El deseo de tener esa pieza única, hecha a mano y con una historia detrás privilegia el trabajo artesanal y viene a compensar el avance de la moda en serie. Por otro lado la valoración de las fibras exóticas se vincula con que fuera del mercado de moda masivo, "la sustentabilidad es la clave" e impacta en la decisión de compra. La buena calidad es tan importante como la trazabilidad del proceso de elaboración de los productos (Lázaro, 2015).

En este sentido, es importante destacar la valorización de las fibras exóticas presentes en el territorio argentino, principalmente la lana de llama y vicuña muy valorizada por países europeos. Marcas argentinas y diseñadores independientes utilizan la vicuña, el lino y el algodón, así como también el cuero producido en territorio argentino en sus colecciones y exportan sus productos a países lejanos.

La marca emergente Pier revaloriza la vestimenta autóctona argentina, utilizando materiales como el cuero y el chaguar. La diseñadora Catalina Pieres da especial valor al trabajo artesanal y la materia prima argentina, así como también cree en una nueva economía en donde el consumidor compre conscientemente y elija un producto que prevalezca en el tiempo y esté hecho en condiciones dignas.

Otro ejemplo es la marca Cúbreme, la cual utiliza en todas sus colecciones lana merino orgánica y lana de llama. Se conjugan la utilización de materiales autóctonos con el ideal de comercio justo y ropa $100 \%$ ecológica (ya que la llama es un animal doméstico y no hay que capturarlo para proceder a su esquila).

Hay diseñadores ya consagrados que han enarbolado la bandera de lo autóctono con una resignificación importante de diseño y que no utilizan la materia prima en estado natural, sino que se basan en técnicas textiles ancestrales como Marcelo Senra, quien utiliza el chaguar, Manuela Rasjido y el barracán y Juana de Arco quien usa el ñandutí.

El diseñador Santos Liendro, va un poco más allá, y comunica perfectamente la cultura argentina andina en una perfecta conjunción entre arte ancestral y proceso de diseño Esto se traduce en accesorios hechos en alpaca, piedras del norte salteño y ónix, y el diseño de tejidos artesanales mixturados con tejidos de manufactura industrial.

\section{La nueva ideología de la moda}

Todo cambio implica una nueva toma de partido y un nuevo comienzo. En este sentido, el cambio de paradigma en la moda ha impulsado un nuevo rol del diseñador de indumentaria y, por lo tanto, nuevas formas de desarrollar prendas de vestir en sintonía con el entorno y enfocada a los usuarios, los cuales se muestran más conscientes y permeables a vivir nuevas experiencias a través de la indumentaria. Anteriormente la mayor preocupación de un creador de moda era la de estar informado y actualizado en relación a las tendencias a nivel macro, poder brindar indumentos con impronta global, lograr una imagen cosmopolita. En la actualidad, con la gran cantidad de información que circula en las redes y en los medios tradicionales de comunicación, las exigencias de los usuarios se han exacerbado, teniendo en cuenta que se encuentran mucho más actualizados e in- 
formados y, por lo tanto, no solo están al tanto de las tendencias, sino que también han aprendido acerca del valor del cuidado medioambiental, del impacto que genera la moda en el entorno y, principalmente, le han dado valor a lo artesanal por sobre lo meramente industrializado.

Es posible determinar que el sistema de la moda, en el inicio del siglo XXI, ha cambiado rotundamente su ideología rectora, pasando de la producción masiva al cuidado de los recursos, dando como resultado lo que hoy se conoce como moda ética en la cual se enmarcan muchos de los proyectos de los diseñadores emergentes argentinos.

Durante la vigencia de la cultura de masas, cuando se impulsaban los consumos a partir de las estrategias que hacían homogéneas las formas, estandarizaban las medidas, vulgarizaban las prendas al simplificarlas y se orientaban a los mercados democráticamente, se desatendían las verdaderas necesidades de las personas. Desde esa situación de búsquedas ficticias, la sociedad actual necesita para su supervivencia hacer cambios tanto en la manera de producir las prendas como en la manera de apropiarse de ellas (Saulquin, 2014, p. 53).

Este cambio ideológico del sistema de la moda ha beneficiado a la gran mayoría de los países latinoamericanos los cuales mantienen una relación muy estrecha con sus recursos naturales, sus técnicas de manufacturación ancestrales y, principalmente, con la producción de fibras textiles orgánicas las cuales son consideradas parte del nuevo lujo del siglo XXI. Asimismo, este nuevo paradigma de la moda, no solo ha puesto en valor lo autóctono y lo artesanal, sino que ha impactado directamente sobre las formas de producción. Al combinar procesos industriales con artesanales en las prendas, los diseñadores de indumentaria han tenido que aprender a conectarse con tiempos de producción más humanos, de esta forma el proceso creativo se vuelve más personalizado y en coherencia con las verdaderas necesidades del usuario. En tal sentido, los tiempos de producción ya no responden a las exigencias de la industria sino de los usuarios, quienes valoran la calidad y el trabajo artesanal que proporciona una historia detrás de cada prenda.

A partir de la revalorización del trabajo artesanal, en el año 2015 se creó en el país el Registro Nacional de Artesanos Textiles de la República Argentina, el cual permite fomentar y registrar la actividad artesanal textil en el territorio, así como también permitir a los artesanos una visibilidad local y mundial de su trabajo.

El ReNATRA es una guía que reúne datos esenciales de cada artesano textil argentino: su nombre propio, la denominación de su emprendimiento, dirección y contacto tecnológico, la actividad artesanal que realiza (hilandería, tejeduría o ambas) y la técnica de tejido que emplea: de punto, de red, a telar, trenzados $\mathrm{u}$ otras técnicas textiles.

Mucha gente trabajó para concretar esta guía, ahora fuente de consulta de acceso público y gratuito. Para generarla, fue vital que se enlazaran múltiples organismos e instituciones (más de 250): del Estado Nacional, gobiernos provinciales y municipales, ONG's y fundaciones (Vallejos, 2015). 
Esta base de datos pretende vincular a los diseñadores de indumentaria y textil con los artesanos para que estos últimos se puedan incorporar al sistema de la moda bajo el criterio de la moda ética y el comercio justo, favoreciendo de esta manera al desarrollo de las economías regionales.

Hay diseñadores argentinos famosos que en las últimas décadas se esforzaron -por convicción y buen gusto- en abrir el camino para que la artesanía textil sea parte de la moda y suba a la pasarela, para luego recorrer el mundo urbano (Vallejos, 2015).

Como bien se ha mencionado anteriormente, el entorno cercano nutre al diseño de indumentaria y textil argentino -y también a nivel latinoamericano- para el desarrollo de emprendimientos con identidad. Asimismo, la tendencia latente acerca del cuidado medioambiental y de los recursos naturales, conjuntamente con la crisis económica, son factores que se conjugan para converger en lo que se conoce actualmente como moda ética, eco moda o moda sustentable. En tal sentido, cabe destacar, que sin crisis no hay cambio, por lo que todos estos nuevos emprendimientos que se han generado a partir de estos nuevos criterios de la moda son producto de las crisis que se afrontan en la actualidad, ya sea medioambiental, social, política, cultural o económica.

A medida que avanza el siglo actual y cumpliendo con su ideología rectora, se afianza cada vez más entre los creadores independientes la importancia del diseño sustentable, con producciones basadas en la ética y en la responsabilidad social. En la actualidad, las formas de producción y reproducción de vestimentas comenzarán a ser prestigiosas en la medida que puedan responder y reflejar la ideología del siglo XXI, contemplando el cuidado de los recursos naturales y de las personas (Saulquin, 2014, p. 90).

En Argentina hay muchos emprendimientos, hoy devenidos en negocios sustentables, que se enmarcan dentro de lo que se conoce como upcycling. Transforman materiales aparentemente de desecho para darles una nueva vida útil y convertirlos en objetos de diseño aportando al cuidado medioambiental y a mejorar la relación que se tiene con el entorno, asimismo ayudando a fomentar y a tomar consciencia acerca del impacto que generan los residuos en el ambiente.

Jessica Pullo apuesta al upcycling para el desarrollo de su marca Biótico. Para la creación de sus prendas apuesta a la reutilización de desechos domésticos urbanos, como sachets de leche, bolsas de nylon, plásticos blancos, descartes textiles y packaging. Asimismo, trabaja colaborativamente con integrantes de la Asociación Laboral para Adultos con Discapacidad Intelectual (Alpad) quienes realizan trabajo sobre los desechos que luego se convertirán en prendas de indumentaria.

La marca de accesorios Modesta también es un caso específico de moda sustentable y upcycling, teniendo en cuenta que apuestan a la reutilización de bolsas plásticas para el desarrollo de carteras, bolsos y sombreros. Cabe destacar que para poder confeccionar un bolso de la marca se necesitan entre 50 y 100 bolsas plásticas. 
Vale la pena mencionar a su vez, a la marca Carro, autodenominada como 'creadora de objetos portantes', es decir que, realizan todo tipo de accesorios contenedores como mochilas, bolsos, morrales y fundas para tablet, notebook, etc. Carro nació en el año 2007, de la mano de las diseñadoras Guillermina Balsells y Silvina Cannito, quienes luego de un viaje a Europa en busca de tendencias, vieron la oportunidad de crear objetos nuevos a partir de prendas en desuso. De este modo, la marca selecciona indumentaria de descarte y reutiliza los textiles rescatados para crear nuevos accesorios portantes.

A través de la deconstrucción de prendas en desuso en combinación con nuevas materialidades trabajan los diseñadores argentinos Mercedes Martinez y Mariano Breccia, dueños de la marca $12 \mathrm{Na}$, la cual se encuentra localizada en Chile pero que tiene proyección tanto en Latinoamérica con en el resto del mundo. Ellos realizan indumentaria mediante la intervención de ropa de segunda mano, retazos de descartes industriales y telas recicladas. De esta manera, crean indumentaria única, original y divertida. Sus prendas se encuentran a la venta en Santiago de Chile, Buenos Aires y Tokio, con posibilidades de expansión hacia otros países asiáticos. Además, realizan vestuario para reconocidos músicos y artistas latinos como El Chavez, los Illia Kuriaky and the Valderramas y los chilenos Fernando Milagros, Gepe y Javiera Mena.

Una significativa macrotendencia a nivel global ha sido mencionada anteriormente: el nuevo lujo. Esta corriente que se impone hace aproximadamente una década y llegó para quedarse, engloba todos los conceptos a los que se ha hecho mención con anterioridad: la sustentabilidad, la atemporalidad, el diseño 'a medida' o customizado y, sobre todo, la conciencia ecológica. "Se trata de volver a la esencia del lujo, a su significado ancestral, la compra meditada, la manufactura artesanal y la belleza de los materiales en su sentido más amplio" (Gardetti, 2013).

Esta nueva búsqueda de lujo, desterrando la antigua concepción de lujo banal, recargado, relativo a precios elevados, privativo para las clases más altas y relacionado con la explotación de materiales sin importar la conciencia medioambiental, es una premisa que se repite en los diseñadores emergentes y resulta su forma de contribuir hacia una nueva moda ética, comprometida y responsable.

Hay un nuevo lujo que no tiene que ver con un producto sofisticado ni carísimo, sino con lo artesanal, en materiales naturales, confeccionado de manera exclusiva, respetando el medio ambiente y las condiciones en que trabajan quienes lo producen. Lo hecho a mano es un lujo. Implica una adquisición meditada, que lo valora; se opone a la compra inconsciente (Vitale, 2016).

Además, en su esencia, el lujo y el desarrollo sustentable van de la mano. Las marcas premium venden productos escasos, dependen de los recursos naturales y la confección artesanal. El lujo promueve una cultura opuesta al usar y tirar nada más lejos del fast fashion; se trata de productos de alta durabilidad y se pasan de generación en generación. Asimismo, requieren de mano de obra calificada, por lo que promueven habilidades especializadas y capacitan a los empleados (Vitale, 2016). 
Si bien todos los ejemplos mencionados anteriormente corresponden con este ideal de nuevo lujo, cabe mencionar algunos otros ejemplos de aplicación de esta nueva mirada sobre el mundo de la moda, las cuales han sido premiadas y galardonadas por su actividad responsable.

En primer lugar, cabe destacar el trabajo de los diseñadores argentinos Ezequiel Gatti, Nazareno El Hom y Alejandro Malgor quienes fabrican zapatillas a partir de residuos de caucho de neumáticos y con retazos de jeans, mamelucos de cortinas desechadas y de otros textiles. La marca Xinca ha sido reconocida como el mejor emprendimiento sustentable del país, y también Naciones Unidas les otorgó el premio GSC 3S (premio mediante el cual se reconoce el trabajo de pequeñas empresas que implementan y promueven innovaciones y prácticas sustentables y socialmente responsables).

En 2013, fue nominada al Premio al mejor desempeño en lujo sustentable en Latinoamérica la diseñadora argentina Lori Carini, creadora de la marca Visceral Argentina, la cual trabaja con mondongo (estómago de vaca) como materia prima fundamental para realizar todo tipo de calzado. Además de la utilización de un material poco convencional, el carácter sustentable y el aprovechamiento de los recursos se encuentra en el proceso de curtido ya que se reutiliza el agua con el que se hace y se utilizan taninos vegetales. Luego de que esos elementos se agotan, se utilizan los desechos como abono.

\section{Conclusiones}

Habrá sido, muy probablemente, en el afán de recuperar algo de todo aquello que la era de Internet, las pantallas líquidas y las redes sociales parecía llevarse puesto por completo a finales de los años noventa, que muchos artistas visuales se inclinaron a explorar telas, hilos, lanas y bordados. Algo del orden de lo íntimo y lo doméstico se juega -lo sabemos y ya ha sido dicho- en este tipo de trabajos, en cuya práctica ordinaria anidaron, de forma anónima y durante siglos, secretos, historias, pequeños universos que hoy, a la -tenue- luz de la vida contemporánea, refulgen como piedras preciosas (Villaro, 2018).

Al tratar de pensar en una identidad argentina de diseño, evocamos el típico traje folclórico, el gaucho y la china, la guarda pampa, el mate y el poncho, siendo tal vez, una forma muy fácil de encasillar un concepto tan vasto y abarcativo como es la identidad.

Aunque es importante decir que no está mal hacer uso de los íconos mencionados anteriormente, el verdadero poder significativo de lo nacional es la creación de una verdadera cultura del diseño argentino y de contemplar en éste una auténtica ventana hacia el mundo con una impronta propia y diferente a las demás regiones del planeta sin perder lo que hace al territorio argentino único e inédito para muchos consumidores de moda.

Hablar de identidad corresponde a varios aspectos de una cultura: incluye también los procesos productivos que se utilizan, las materialidades presentes autóctonas y de cada región, la relación que la sociedad tiene con el medio ambiente, la forma de creación de tejidos y procesos de diseño, y, también la personalidad de sus habitantes tanto como los procesos económicos y políticos que atraviesan al país. 
Es común oír en los dichos de diseñadores argentinos que somos hijos de la crisis, y esta es una gran verdad, pero también una cualidad significativa y sólida para otorgar una personalidad resiliente y adaptable a cambios bruscos. Ésta veta, además de las riquezas naturales que posee el territorio argentino, es la que destaca a los creadores de la región en relación a los diseñadores de otras partes del mundo.

Un diseñador de moda no es una isla, sino que se nutre del medio que lo rodea. Por lo tanto, no es indiferente a las tendencias ni a los sucesos que acontecen de forma global, pero, en vez de realizar la antigua copia de tendencias europeas, la nueva generación de diseñadores, analiza y entiende las tendencias latentes para luego seleccionar aquello que se ajusta a su identidad aplicada a su personalidad como creador. De manera que, al momento de generar una identidad nacional, también se busca que la misma sea aplicable en diferentes partes del mundo. Es decir, que sea aceptada y posible de usar en cualquier lugar sin dejar de contar la historia detrás de la prenda.

En cada pieza se comunica una marcada territorialidad, en el cual se manifiesta un acentuado relato que interpreta la riqueza del medio que los rodea, los preciosos recursos naturales con los que se cuenta y el patrimonio cultural que el arte ancestral otorga. A todo esto, es relevante adicionar la genuina preocupación por el medio ambiente y el cuidado de los recursos, de manera que a la valorización de los procesos artesanales (ya sea en materia de tejidos, teñidos y/o utilización de materias primas naturales u orgánicas) se le suma la sustentabilidad y el comercio justo como valores insoslayables. Aquí nace el nuevo lujo, el consumo consciente dando como resultado la consumación de una moda completamente ética.

Para finalizar, es importante destacar que los diseñadores emergentes argentinos y latinoamericanos analizan las opciones que tienen a su alcance, investigan y buscan nuevos materiales para su aprovechamiento, analizan su entorno y medio ambiente, soslayan crisis y vaivenes económicos, buscan incorporar la ética y el comercio justo al mundo de la moda, pero, el rasgo más significativo es que son resilientes y no se detienen, buscan continuamente nuevos horizontes.

\section{Bibliografía}

Brovia, F. (2016). Vestir a consciencia, el nuevo lujo. Buenos Aires: Diario Clarín. Recuperado el 11702/2018. Disponible en: https://www.clarin.com/entremujeres/moda/fashion-tips/ vestir-consciencia-nuevo-lujo_0_rykVv0fHe.html

Colzani, G. (2014). Tejiendo identidades latinoamericanas. Cuadernos del Centro de Estudios de Diseño y Comunicación No 47. Buenos Aires: Universidad de Palermo. Disponible en: http://fido.palermo.edu/servicios_dyc/publicacionesdc/archivos/459_libro.pdf

Gadea Lara, T. (2017). Empresas "ecofriendly" que apuestan por el diseño sustentable. Recuperado el 30/01/2018. Disponible en: https://www.lanacion.com.ar/2015871-empresasecofriendly-que-apuestan-por-el-diseno-sustentable

Gardetti, M. (2013). Nuevo lujo para uno y lo demás. Citado en: Tapia, P. (2013) Nuevo lujo para uno y los demás. Recuperado el 11/02/2018. Disponible en: https://www.clarin. com/mujer/Nuevo-lujo_0_rkHXYDMoPmg.html 
Guerrero Arias, P. (2002). La cultura. Estrategias conceptuales para entender la identidad, diversidad, la alteridad y la diferencia. Quito: Abya-Yala. Disponible en: http://digital repository.unm.edu/abya_yala/10/

Godart, F. (2012). Sociología de la moda. Buenos Aires: Edhasa.

Lázaro, A. (2015). Fibras naturales argentinas. Recuperado el 07/02/2018. Disponible en: https:/lamodaenserio.com/fibras-naturales-argentinas/

Maurello, E. (2016). Carro, trabajando el upcycling desde Argentina. Recuperado el 11/02/2018. Disponible en: https://www.slowfashionnext.com/blog/2016/07/27/carro-trabajando-elupcycling-desde-argentina/

Mercado de Industrias Creativas de Argentina (2017). Dis.Glosario federal del diseño argentino. Santa Fe: Mercado de Industrias Creativas de Argentina (MICA). Consultado el 26/01/2018. Disponible en https://mica.cultura.gob.ar/wp-content/uploads/sites/ 10/2017/10/DisglosarioWeb-MCN.pdf

Mon, L. (2013). Diseño en Argentina. Hacia la construcción de nuevos paradigmas. Cuadernos del Centro de Estudios de Diseño y Comunicación $N^{\circ} 44$. Buenos Aires: Universidad de Palermo.

Montenegro, T. (2012). $12 \mathrm{Na}$, el reciclaje como medio de expresión. Revista Dínamo. Recuperado el 11/02/2018. Disponible en: http://www.revistadinamo.com/?p=2562

Organización Internacional para las Migraciones (2008). Perfil migratorio de Argentina. Buenos Aires: Organización Internacional para las Migraciones. Consultado el 10/01/2018. Disponible en http://www.mininterior.gov.ar/provincias/archivos_prv25/6-\%20Perfil _Migratorio_de_la_Argentina.pdf

Saulquin, S. (2009). Indumentaria. Diario La Nación. Recuperado el 30/01/2018. Disponible en: http://www.lanacion.com.ar/1217023-indumentaria

Saulquin, S. (2014). Política de las apariencias. Paidós: Buenos Aires.

Vallejos, M. (2015). Que se vea, aqui y afuera: la identidad de los artesanos textiles. Recuperado el 07/02/2018. Disponible en: https://lamodaenserio.com/la-identidad-de-losartesanos-textiles/

Villaro, J. (2018). El territorio que cabe en una trama. Buenos Aires: Revista Ñ. Recuperado el 11/02/2018. Disponible en: https://www.clarin.com/revista-enie/arte/territoriotrama_0_BJjpOs1LM.html

Vitale, S. (2016). Cómo es el lujo sustentable. Buenos Aires: Diario La nación. Recuperado el 11/02/2018. Disponible en: https://www.lanacion.com.ar/1899992-como-es-el-lujosustentable

Abstract: Talking about the current worldwide situation is highly contradictory. On the one hand, the massification that globalization produces is undeniable, therefore, the existing unity between the different countries and the new forms of communication and access to information make possible the instantaneous connection at the global level; while on the other hand there is a huge need for differentiation and, in this sense, the return to roots and exploration within the territory of origin have given a new sense to fashion and has given a new role to fashion designers. The search for a marked identity is generated from 
the claim of ancestral textile techniques, textile experimentation, sustainability, slow fashion and upcycling, nationalism to the surface, functionality of the hand with intelligent textiles, among other trends in the design of dress.

Keywords: fashion - trends - Clothing design - conscious fashion - sustainability - new luxury - resilience.

Resumo: Falar sobre a situação em que o mundo é atualmente é contraditório. Por um lado, a massificação que a globalização produz é inegável, portanto, a unidade existente entre os diferentes países e as novas formas de comunicação e acesso à informação possibilitam a conexão instantânea em nível global; enquanto, por outro lado, há uma enorme necessidade de diferenciação e, nesse sentido, o retorno às raízes e a exploração dentro do território de origem deram um novo sentido à moda e deram um novo papel ao designer de roupas. A busca por uma identidade marcante é gerada a partir da reivindicação de técnicas têxteis ancestrais, experimentação têxtil, sustentabilidade, slow fashion e upcycling, nacionalismo à superfície, funcionalidade da mão com tecidos inteligentes, entre outras tendências no design de vestuário.

Palavras-chave: Moda - tendências - Design de roupas - moda consciente - sustentabilidade - novo luxo - resiliência.

[Las traducciones de los abstracts fueron supervisadas por el autor de cada artículo] 